\title{
Persone come noi: intervista a Maricia Roccaro
}

Giornale di Tecniche Nefrologiche e Dialitiche 2018, Vol. 30(I) 63-65

(C) The Author(s) 2018

Reprints and permissions:

sagepub.co.uk/journalsPermissions.nav

DOI: $10.1177 / 0394936218766532$

journals.sagepub.com/home/gtn

(3SAGE

La malattia di Fabry e AIAF Onlus

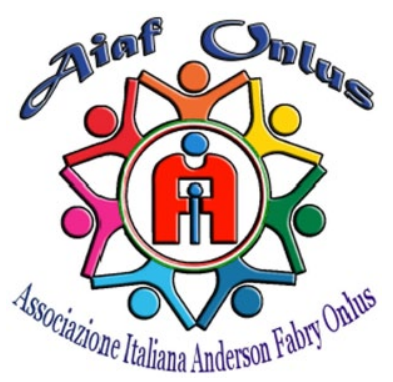

Vi raccontiamo la singolare testimonianza di Maricia Roccaro, di Catania, nefrologo da circa 20 anni, che si occupa da molto tempo di pazienti in dialisi e da anni dirige un centro dialisi in Sicilia.

\section{Maricia, com'è avvenuto il tuo primo "contatto" con la Malattia di Anderson-Fabry?}

Ognuno di noi pensa che la propria storia sia unica... oltre ad essere unica è certamente una storia in cui, ritengo, qualcosa di soprannaturale si sia davvero concentrato su di me, nel modo più benevolo che possa esistere.

Il tutto ha inizio in un giorno come tanti: era una mattina del mese di gennaio 2016, quando un giovane informatore scientifico, mai conosciuto prima, bussa alla mia porta e mi chiede un appuntamento. Lo accolgo da lì a poco.

Il suo entusiasmo, assieme alla sua professionalità mi colpiscono positivamente; mi parla di una malattia rara, che avrei dovuto ben conoscere, in quanto malattia che vede come organi bersaglio soprattutto i reni ma anche il cuore e il cervello. Nonostante io mi fossi interfacciata tante volte con altri informatori scientifici, confesso di non avere mai prestato particolare attenzione a tale malattia, perché pensavo che, essendo rara, non avrei mai incontrato un paziente che ne fosse affetto. Essendo oberata da tanto lavoro, non avevo certo tempo per ascoltare, o addirittura approfondire, tale malattia.

\section{Quindi non ne avevi mai sentito parlare prima?}

La malattia di Anderson-Fabry era una malattia realmente poco nota e nonostante io avessi un bel poster proprio nella mia stanza, di fatto a quel poster non avevo mai prestato attenzione!

\section{In che modo ti sei lasciata coinvolgere, quel giorno, dall'informatore scientifico?}

Quel giorno di gennaio, io probabilmente ero particolarmente calma e recettiva tanto che non mi feci coinvolgere superficialmente dall'informatore scientifico, che mi proponeva un'opportunità di diagnosi ai miei pazienti dializzati.

Potere fare diagnosi, anche se i pazienti dializzati avevano ormai perduto la funzione renale, avrebbe permesso di verificare se un altro familiare ne fosse affetto e a quel punto per i familiari avrebbe consentito una grande opportunità di bloccare la malattia con una terapia specifica.

Decisi pertanto di organizzarmi con l'informatore scientifico, per effettuare il test genetico a tutti i miei pazienti dializzati.

\section{E a quel punto, però, hai preso una decisione molto particolare.... Ce la vuoi raccontare tu?}

Certo. Non so per quale arcano motivo chiesi di fare anche io il test. "Arcano motivo" perché stavo male da un po', ma il malessere era talmente sfumato e comprendeva astenia persistente (da sempre) ed il forte timore (dall'anno prima), di essere affetta da sclerosi multipla, poiché per circa 20 giorni ne avevo percepito forti somiglianze cliniche. E fu così, che da lì a pochi giorni, tutti i miei pazienti (compresa io) effettuammo il test.

Per la verità, fui così coinvolta da quell'informatore scientifico, che decisi di far eseguire il test anche ad altri miei pazienti che ancora non facevano dialisi. 


\section{E poi, cosa è accaduto nei giorni successivi?}

Devo ammettere che attendevo con curiosità l'esito dei test: pensavo a chi dei miei pazienti potesse avere tale malattia, sia ricostruendone la storia clinica, sia ripensando all'anamnesi familiare.

Iniziai anche a parlare della Malattia di Anderson-Fabry ai miei collaboratori.

\section{Cos'è accaduto quando sono arrivati $i$ risultati dei test}

Dopo circa un mese e mezzo, ricevetti la telefonata dell'informatore scientifico e la comunicazione che tra $\mathrm{i}$ miei pazienti c'era un paziente affetto da Fabry!

Con trepidazione, aprii i file relativi ai risultati dei test, mentre facevo alcune ipotesi di diagnosi pensando a 6 pazienti in particolare... ero quasi certa che il paziente affetto da Fabry fosse tra loro.

Con mio sommo sgomento, però, scoprii che NESSUNO dei miei pazienti era affetto da Malattia di AndersonFabry... perché la persona ad avere la malattia ero io!!!!

\section{Improvvisamente, quindi, sei passata dal ruolo di "clinico", al ruolo di "paziente"... Come hai vissuto questo momento?}

In un primo momento sono proprio scoppiata a piangere come una bambina... mi sembrava una beffa del destino, ho pensato quanto poco sapessi di questa malattia, nonostante fossi medico e specialista di una branca, la nefrologia, che tanto aveva a che fare con la malattia di Fabry. In quei frangenti "acuti" però il pensiero era rivolto a me, alla mie condizioni cliniche... Cercavo con grande confusione emotiva di ricostruire la tracciabilità sui miei sintomi, cercavo di concentrarmi sulla mia storia familiare... ma in cima ai miei pensieri, in modo assolutamente preponderante e crescente era il pensiero rivolto ai miei due bambini di 8 e 10 anni... Che vita mi si preparava?

Mi affrettai a concentrare la mia attenzione su ciò che recitava il referto circa una correlazione non con un'eventuale compromissione renale, bensì con una compromissione neurologica e cardiologica. $\mathrm{Fu}$ così che ogni mio sintomo, assumeva un ruolo di assoluta rilevanza: la mia astenia così gravosa, a volte davvero invalidante, $\mathrm{i}$ miei dolori "neuropatici", specie alle gambe, la mia percezione crescente di avere un udito poco fine... TUTTO in quel momento assumeva una fisionomia diversa e come le tessere di un puzzle tutto trovava il suo giusto posto. Feci immediatamente una RM dell'encefalo che evidenziò una pregressa ischemia!!!!
Da lì a poco, con i test successivi, appresi anche la notizia che entrambi i miei bambini avevano ereditato la malattia.

\section{Trattandosi di una malattia genetica rara, immagino che tu ti sia rivolta ad un centro specializzato e territorialmente vicino al tuo luogo di residenza. È stata una cosa semplice?}

Non avevo alterazioni enzimatiche: ancora l'attività enzimatica era fortunatamente efficiente, ma la neurologa che mi visitò con un'accuratezza maniacale mi disse con assoluta fermezza che avevo necessità di iniziare il trattamento farmacologico.

Iniziai personalmente un percorso diagnostico presso l'Azienda Ospedaliero Universitaria "Policlinico Vittorio Emanuele" di Catania, dove fui sottoposta ad un'accurata valutazione clinica polispecialistica, ma al momento della prescrizione della terapia farmacologica scoprii, con sommo sgomento, che era stata sospesa l'autorizzazione alla prescrizione del farmaco al Policlinico di Catania: oltre alla sottoscritta, a causa di questi problemi burocratici, non era stato possibile prescrivere il farmaco anche ad altri 5 pazienti!

Nonostante io fossi medico, ho brancolato nella confusione per tanti mesi. Mesi nei quali anche problematiche personali si sono certamente sommate, ma oggettivamente, per chi sta male ed ha bisogno di essere guidato dal punto di vista della gestione della propria malattia rara, è stata davvero un'odissea arrivare a comprendere il percorso più adeguato per la gestione della propria patologia.

\section{Quindi, mi sembra di capire che la burocrazia, in questo caso, abbia complicato ulteriormente il percorso del paziente per ricevere una terapia, anziché semplificarlo... Come hai deciso di affrontare la situazione per poter iniziare la terapia necessaria?}

Dopo un ulteriore lungo peregrinare, fatto da approcci telefonici, ricerche estenuanti, confronti anche con altri malati, sono approdata finalmente al Centro Regionale di Pediatria Genetica e Malattie Rare presso l'Università Magna Graecia di Catanzaro, in Calabria, che è certamente uno dei centri italiani di eccellenza, che ha accolto me e i miei bambini con competenza, umanità e professionalità. Lì avrei potuto iniziare la terapia enzimatica sostitutiva dopo aver completato tutti gli accertamenti e approfondimenti clinici. Ero tuttavia consapevole che avrei dovuto affrontare presto un nuovo problema, rappresentato dal 
dover affrontare un viaggio in aereo ogni 14 giorni, almeno per i primi mesi, per ricevere la terapia. Per me sarebbe stato veramente troppo complicato riuscire a conciliare la vita familiare, il lavoro (per il quale sono spesso in viaggio) e una terapia al di fuori della mia regione. Ero preoccupata e temevo di non riuscire a gestire questa situazione.

Nel mese di novembre, però, fui invitata dalla mia neurologa, Dottoressa Antonia Nucera, a partecipare ad un convegno di aggiornamento medico-scientifico sulla Malattia di Anderson-Fabry, al quale avrebbero partecipato medici specialisti provenienti da tutta Italia. Colsi questa opportunità, ritenendo potesse essere utile approfondire ulteriormente la conoscenza di questa malattia, anche e soprattutto per la mia professione di nefrologo. In quell'occasione, ebbi modo di conoscere il Prof. Antonino Tuttolomondo dell'Azienda Ospedaliera Universitaria Policlinico "Paolo Giaccone" di Palermo, che avvicinai per raccontargli la mia incredibile storia e tutte le difficoltà incontrate. In lui trovai una nuova rassicurazione, perché si rese disponibile a fissare un appuntamento al Policlinico di Palermo per valutare la mia situazione clinica. Accolsi con sollievo questa nuova opportunità, che poteva evitarmi tutte le preoccupazioni di affrontare lunghi viaggi fuori regione per essere curata. In brevissimo tempo, fui accolta a Palermo per una prima visita e, dopo essere stata sottoposta a tutti i necessari accertamenti, ricevetti la notizia che avrei potuto iniziare la terapia enzimatica sostitutiva presso il Policlinico.

Ho finalmente ricevuto la mia prima infusione il 28 dicembre 2017 e la prospettiva sarà quella di poter proseguire, tra qualche mese, la terapia al domicilio, ma solo se non si verificheranno effetti collaterali per un certo numero di infusioni, come stabilito dai protocolli di somministrazione di questo farmaco. Se ciò sarà possibile, sarebbe davvero un'ottima opportunità per me: mi eviterebbe il disagio degli spostamenti da Catania a Palermo e mi permetterebbe di conciliare al meglio la mia vita lavorativa, familiare e la gestione della mia malattia.

Ringrazio Maricia, per averci raccontato la sua testimonianza, molto singolare, che ci ha permesso di mettere in luce alcune problematiche in cui si è imbattuta: da una parte la difficoltà nell'interpretare tutti i sintomi da parte di chi non conosce bene la malattia di Anderson-Fabry e di collegarli tra loro per arrivare ad un sospetto diagnostico o alla diagnosi (e il suo duplice ruolo di medico e paziente ne è un esempio paradossale); dall'altra, le tante difficoltà che incontrano i pazienti nel districarsi tra la burocrazia, che alle volte può complicare ulteriormente la convivenza con una malattia rara, fino a costringerli ad intraprendere lunghi viaggi con dispendio di energie e risorse economiche, per poter accedere alle terapie. Si tratta di due problematiche su cui i vari portatori di interesse potranno indirizzare le proprie attenzioni, in sinergia tra loro, per cercare delle possibili soluzioni, con l'obiettivo di contribuire a migliorare la qualità della vita dei pazienti.

Intervista raccolta da Stefania Tobaldini Presidente AIAF Onlus 